\title{
Implementing Adaptive Vectorial Centroid in Bayesian Logistic Regression for Interval Type-2 Fuzzy Sets
}

\author{
Ku Muhammad Naim Ku Khalif ${ }^{1,1}$, Alexander Gegov ${ }^{1}$ \\ ${ }^{1}$ School of Computing, University of Portsmouth, \\ Buckingham Building, Lion Terrace, Portsmouth PO1 3HE, United Kingdom \\ \{muhammad.khalif, alexander.gegov\}@port.ac.uk
}

\begin{abstract}
A prior distributions in standard Bayesian knowledge are assumed to be classical probability distribution. It is required to representable those probabilities of fuzzy events based on Bayesian knowledge. Propelled by such real applications, in this research study, the theoretical foundations of Vectorial Centroid of interval type-2 fuzzy set with Bayesian logistic regression is introduced. As opposed of utilising type-1 fuzzy set, type-2 fuzzy set is recommended based on the involvement of uncertainty quantity. It additionally highlights the association of fuzzy sets with Bayesian logistic regression permits the use of fuzzy attributes by considering the need of human intuition in data analysis. It may be worth including here that this proposed methodology then applied for BUPA liver-disorder dataset and validated theoretically and empirically.
\end{abstract}

Keywords: Machine learning, Bayesian logistic regression, interval type-2 fuzzy set, defuzzification, Vectorial Centroid, human intuition, uncertainty.

\section{Introduction}

Through the most recent decade, uncertainty problems are common issue in complex systems. In describing uncertainty, a lot of techniques have drawn the attentions of researchers and applied scientist over last decade. Decisions are made based on information given which known as data. However, information about decision is always uncertain. In real-world phenomena, the uncertain information may consist of randomness, vagueness and fuzziness. Machine learning has always been considered

${ }^{1}$ Corresponding author. A. Gegov, School of Computing, University of Portsmouth, Portsmouth PO1 3HE, UK. Tel.: +44 239284 6381; E-mail: alexander.gegov@port.ac.uk. 
as an integral part of the field of artificial intelligence. In artificial intelligence research area, the main problems that always arise are: how to reason uncertain information precisely and: how to reason using uncertain information [1]. Machine learning is certainly one of the most significant subfields of modern artificial intelligence. In recent years, machine learning systems have been adopted standard framework to deal with imprecision in data analysis.

In dealing with imprecise data, type-1 fuzzy set is used as a unique tool to erase these imprecision appropriately. Uncertainty is closely connected with probability, which establishes the formal framework in machine learning systems. Uncertainty and fuzziness are very prominent phenomena in science and engineering applications, where most of researchers nowadays are often used type-1 fuzzy for their case studies. Some of the input data sets, we can't describe straight away or objectively because they have different interpretations and very subjective. Even, type-1 fuzzy set can't tackle the uncertainty component completely because the degree of membership grade of type-1 fuzzy set is focusing on imprecision only. Type-2 fuzzy set is capable to deal with uncertainty or approximate reasoning, especiallly for the machine learning systems with a mathematical model that is difficult to derive. [2] claim that type-1 fuzzy set only describe imprecise not uncertainty. On particular motivation for the further interest in type-1 fuzzy set that its' provide a better scope for modelling uncertainty than type-1 fuzzy set [3].

Zadeh [4] introduced fuzzy set theory in representing vagueness or imprecision in a mathematical approach. In order to do so, the foremost motivation of using fuzzy set shows its ability in appropriately dealing with imprecise numerical quantities and subjective preferences of decision makers [5]. Fuzzy numbers are represented as possibility distribution where most of the real-world phenomena exist in nature are fuzzy rather than probabilistic or deterministic [6]. It was specifically designed to mathematically represent to randomness and also provide formalised tools for dealing with imprecision essential to many real problems nowadays. Technologies nowadays have been developed in fuzzy set that have potential to support all of the steps that encompass a process of model orientation and knowledge discovery. In particular, fuzzy set theory can be used in data analysis to model vague data in terms of fuzzy set. These are some contributions that fuzzy set can assist machine learning which are: 1) graduality; 2) granulity; 3) interpretability; 4) robustness; 5) representation of uncertainty; 6) incorporation of background knowledge and; 7) aggregation, combination and information fusion [7]. In particular, fuzzy set theory can already be used in in the data selection or data processing. For analysing the fuzzy data, there are two different ways: 1) fuzzifying the mapping from data to model and: 2) embed the data into more complex mathematical spaces, like fuzzy metric spaces.

Type-2 fuzzy set notion was introduced by Zadeh [8] as an extension of the type-1 fuzzy set. In accordance to [9], type-2 fuzzy set can be considered as fuzzy membership function where the membership value for each element in type- 2 is a fuzzy set in $[0,1]$, different with type-1 fuzzy set where the membership value is in crisp condition between $[0,1]$. The interval type-2 fuzzy set is extensively used in type-2 fuzzy set family in many practical science and engineering areas [10]. The participation of higher level uncertainty of type-2 fuzzy set compared to type-1, provide additional degrees of freedom to represent the uncertainty and the fuzziness of real-world problems. Uncertainty can be divided into two types which are inter and 
intra personal uncertainties, in improvising the representation of type-1 fuzzy set in the literature of fuzzy sets. This is also supported by [11] where there are supposedly two kinds of uncertainties that are related to linguistic characteristics namely intrapersonal uncertainty and inter-personal uncertainty, In fact, a lot of experts have applied interval type-2 fuzzy set in machine learning systems analysis. Due to implementing interval type-2 fuzzy set in real-world problems, the way to handle is different and much more complex compared to type-1 fuzzy set. The contribution of centroid of type-2 fuzzy set still now commonly used uncertainty measure for modelling problems. Interval type-2 fuzzy set offer an approach to handle knowledge uncertainty in machine learning systems.

Defuzzification plays an important role in the performance of fuzzy system's modelling techniques [12]. Generally, defuzzification process is guided by the output fuzzy subset that one value would be the selected as a single crisp value as the system output. There are variation defuzzification methods have largely developed. Nevertheless, they have difference performances in difference applications and there is a general method can satisfactory performance in all conditions [13]. The centroid defuzzification methods of fuzzy numbers have been explored for the last few decades that commonly used and have been applied in various discipline areas. The computation complexity of type- 2 fuzzy set is very problematic to handle into practical applications because of characterised by their footprint of uncertainty (FOU) [14]. Two typical directions in computing type-2 fuzzy set intensively: 1) typereduction [9], [14], [15] and; 2) direct defizzification [16]. Most experts applied typereduction methods in handling the complexity of type-2 fuzzy set by finding the equivalent type-1 fuzzy set. Though, direct defuzzification computational for type- 2 fuzzy set is still under study.

The possibility mean value for interval sets was introduced by [17] where the notations of lower possibilistic and upper possibilistic mean values are defined the interval-valued possibilistic mean. From probabilistic viewpoint, the possibility mean value of fuzzy sets can be represented as expected values which is same function as direct defuzzification method where it does not need type-reduction or conversion stage into type-1 fuzzy to get the outputs. [16] extend the concept of [17] about possibility mean value of type-1 fuzzy set which introduce the lower and upper possibility mean value for interval type-2 fuzzy set. In this paper, the comparative simulation results between the proposed of the extension of Vectorial Centroid [18] and possibility mean value proposed by [16] method for interval type-2 fuzzy set, where in some cases it will give illogical and irrational results that inconsistent with human intuition. This method can't cater all possible cases of interval type- 2 fuzzy set properly since some of the results are dispersed far away from the closed interval bounded by the expectations calculated from its upper and lower distribution functions.

Due to growths in computational capability and technology development, data are being generated for understanding details real-world problems in health nowadays that associated with clinic test, diseases, disorder, genetic cases and so forth [19]. Yet, with the availability of large datasets become the essential challenges of a new methods of statistical analysis and modelling. Logistic regression model is one of machine learning systems that used in handling these problems with high-dimensional data. The dataset that represents binary dependent attribute where it uses logit 
transform to predict probabilities directly. Logistic regression is a model-based approach to mapping observers' distribution. When applied within Bayesian setting, logistic regression provides a useful platform for integrating expert knowledge, in the form of a prior, with empirical data [20]. Probability is a complete with parametric models that let us characterised random uncertainty [10]. Prior knowledge can be amalgamated into Bayesian logistic regression and the method is computationally efficient.

Issues with respects to representation capability of fuzzy sets in machine learning systems on uncertainty become one of the significant problems in decision making environments. The aim of the present paper is to illustrate the extension of Vectorial Centroid [18] method for interval type-2 fuzzy set that consider the illustration of Bayesian algorithm about the parameters of a logistic regression model. Aiming at the problems pointed out above, new centroid defuzzification for interval type-2 fuzzy set is proposed that easy to understand, more flexible and more intelligent compared to existing methods. The proposed method also considers the need of human intuition and gives logical results while dealing with machine learning systems. In this research study, classification dataset with binary dependent attribute is used. The observations in this dataset, we worked on "BUPA liver-disorder" that were sampled by BUPA Medical Research Ltd. There are 7 attributes that consist of six independent attributes and one binary dependent attribute. The BUPA liverdisorder dataset represents blood tests indicating a property of liver disorders that may increase from excessive alcohol consumption.

The remainder of this paper is organised as follows: Section II introduces the concepts of type-2 fuzzy set, interval type-2 fuzzy set, centroid method that proposed by [16] and Bayesian logistic regression. Section III views the proposed new centroid method for interval type-2 fuzzy sets using Vectorial Centroid method. Section IV illustrates the implementation of proposed method with Bayesian logistic regression in BUPA liver-disorder and compares the results with [16] method. Section V summarises the main results and draws conclusion.

\section{Preliminaries}

\subsection{Interval Type-2 Fuzzy Set}

Definition 1: A type-2 fuzzy set $\tilde{A}$ in the universe of discourse $X$ represented by the type-2 membership function $\mu$. If all $\mu_{\tilde{A}}(x, u)=1$, then $\tilde{A}$ is called an interval type2 fuzzy set. An interval type-2 fuzzy set can be considered as a special case type- 2 fuzzy set, denoted as follows [5]:

$$
\approx \tilde{A}=\int_{x \in X} \int_{u \in J_{x}} 1 /(x, u), \text { where } J_{x} \subseteq[0,1]
$$


Definition 2: The upper and lower membership function of an interval type-2 fuzzy set are type-1 fuzzy set membership functions, respectively. A trapezoidal interval type-2 fuzzy set can be represented by $\widetilde{\tilde{A}}_{i}=\left(\tilde{A}_{i}^{U}, \tilde{A}_{i}^{L}\right)=\left(\left(a_{i 1}^{U}, a_{i 2}^{U}, a_{i 3}^{U}, a_{i 4}^{U} ; H_{1}\left(\tilde{A}_{i}^{U}\right), \tilde{H}_{2}\left(\tilde{A}_{i}^{L}\right)\right),\left(a_{i 1}^{L}, a_{i 2}^{L}, a_{i 3}^{L}, a_{i 4}^{L} ; H_{1}\left(\tilde{A}_{i}^{L}\right), H_{2}\left(\tilde{A}_{i}^{L}\right)\right)\right)$ where can be shown in Fig. 1 [16]. The $\tilde{A}_{i}^{U}$ and $\tilde{A}_{i}^{L}$ are type-1 fuzzy sets, $a_{i 1}^{U}, a_{i 2}^{U}, a_{i 3}^{U}, a_{i 4}^{U}, a_{i 1}^{L}, a_{i 2}^{L}, a_{i 3}^{L}$ and $a_{i 4}^{L}$ are the reference points of the interval type-2 fuzzy set $\tilde{A}, H_{j}\left(\tilde{A}_{i}^{U}\right)$ denote the membership value of the element $a_{i(j+1)}^{U}$ in the upper trapezoidal membership function $\tilde{A}_{i}^{U}, 1 \leq j \leq 2, H_{j}\left(\tilde{A}_{i}^{L}\right)$ denotes the membership value of the element $a_{i(j+1)}^{L}$ in the lower trapezoidal membership function $\tilde{A}_{i}^{L}, \quad 1 \leq j \leq 2, \quad$ and $\quad$ for $\quad H_{1}\left(\tilde{A}_{i}^{U}\right) \in[0,1], \quad H_{2}\left(\tilde{A}_{i}^{U}\right) \in[0,1]$, $H_{1}\left(\tilde{A}_{i}^{L}\right) \in[0,1], H_{2}\left(\tilde{A}_{i}^{L}\right) \in[0,1]$ and $1 \leq i \leq n, H_{2}\left(\tilde{A}_{i}^{U}\right) \in[0,1][5]$.

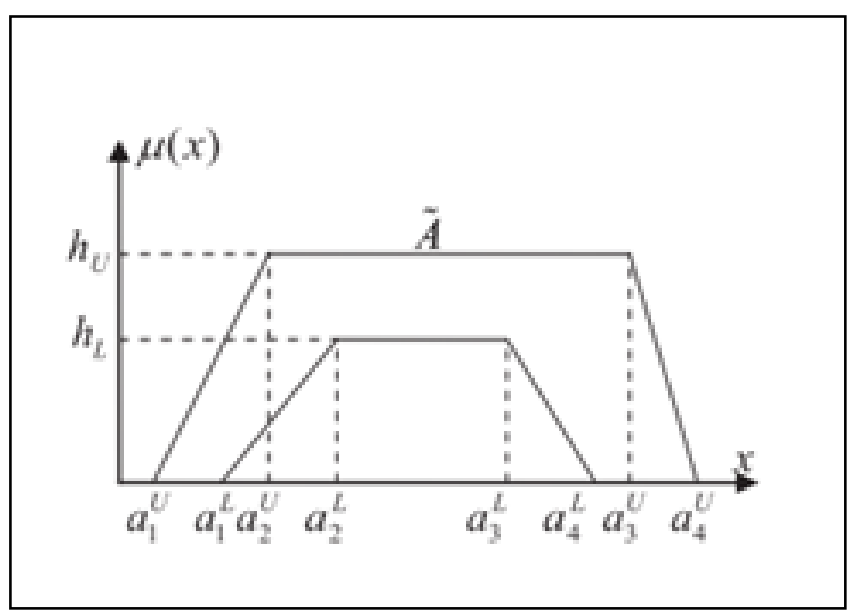

Fig. 1. The upper trapezoidal membership function $\tilde{A}_{i}^{U}$ and lower trapezoidal membership function $\tilde{A}_{i}^{L}$ of interval type-2 fuzzy set.

\subsection{Bayesian Logistic Regression}

The principal of Bayesian inference for logistic regression analyses follows the typical pattern for Bayesian analysis [22]:

1. Write down the likelihood function of the data

2. Form a prior distribution over all unidentified parameters 
3. Find posterior distribution using Bayes theorem over all parameters

Likelihood function: the likelihood contribution from the $i^{\text {th }}$ subject is binomial

$$
\text { likelihood } \left._{i}=\pi\left(x_{i}\right)^{y_{i}}\left(1-\pi\left(x_{i}\right)\right)^{\left(1-y_{i}\right.}\right)
$$

where $\pi\left(x_{i}\right)$ represents the probability of the event for subject $i$, which has covariate vector $x_{i}$ and $y_{i}$ specifies the liver-disorder $y_{i}=1$, or liver-normal $y_{i}=2$ of the event for the subject. Logistic regression is denoted as

$$
\pi(x)=\frac{e^{\beta_{0}+\beta_{1} X_{i}+\ldots+\beta_{p} X_{p}}}{1+e^{\beta_{0}+\beta_{1} X_{i}+\ldots+\beta_{p} X_{p}}}
$$

So the likelihood from the $i^{\text {th }}$ subject is

$$
\begin{aligned}
\text { likelihood }_{i} & =\left(\frac{e^{\beta_{0}+\beta_{1} X_{i 1}+\ldots+\beta_{p} X_{\text {ip }}}}{1+e^{\beta_{0}+\beta_{1} X_{i} 1+\ldots+\beta_{p} X_{\text {ip }}}}\right)^{y_{i}}\left(1-\frac{e^{\beta_{0}+\beta_{1} X_{i 1}+\ldots+\beta_{p} X_{\text {ip }}}}{1+e^{\beta_{0}+\beta_{1} X_{i 1}+\ldots+\beta_{p} X_{i p}}}\right)^{\left(1-y_{i}\right)} \\
\text { likelihood }_{i} & =\prod_{i=1}^{n}\left[\left(\frac{e^{\beta_{0}+\beta_{1} X_{i 1}+\ldots+\beta_{p} X_{\text {ip }}}}{1+e^{\beta_{0}+\beta_{1} X_{i} 1+\ldots+\beta_{p} X_{\text {ip }}}}\right)^{y_{i}}\left(1-\frac{e^{\beta_{0}+\beta_{1} X_{i 1}+\ldots+\beta_{p} X_{\text {ip }}}}{1+e^{\beta_{0}+\beta_{1} X_{i} 1+\ldots+\beta_{p} X_{\text {ip }}}}\right)^{\left(1-y_{i}\right)}\right]
\end{aligned}
$$

Prior distribution: in general, any prior distribution can be used, depending on the available prior information.

$$
\beta_{j} \sim \operatorname{Normal}\left(c_{j}, \sigma_{j}^{2}\right)
$$

The most common choice for $c$ is zero, and $\sigma$ is usually chosen to be large enough to be considered as non-informative in the range from $\sigma=10$ to $\sigma=100$.

Posterior distribution via Bayes theorem: the posterior distribution is divided by multiplying the prior distribution over all parameter by the full likelihood function, so that

$$
\text { Posterior }=\prod_{i=1}^{n}\left[\left(\frac{e^{\beta_{0}+\beta_{1} X_{i 1}+\ldots+\beta_{p} X_{\dot{\varphi}}}}{1+e^{\beta_{0}+\beta_{1} X_{i} 1+\ldots+\beta_{p} X_{\dot{\varphi}}}}\right)^{y_{i}}\left(1-\frac{e^{\beta_{0}+\beta_{1} X_{i 1}+\ldots+\beta_{p} X_{\dot{\varphi}}}}{1+e^{\beta_{0}+\beta_{1} X_{i} 1+\ldots+\beta_{p} X_{\dot{\varphi}}}}\right)^{\left(1-y_{i}\right)}\right] \times \coprod_{j=0}^{p} \frac{1}{\sqrt{2 \pi \sigma}} \exp \left\{-\frac{1}{2}\left(\frac{\beta_{j}-c_{j}}{\sigma_{j}}\right)^{2}\right\}
$$

The latter part of the above expression being recognised as normal distribution for the $\beta$ parameters. For liver-disorder classification problem, $p\left(y=1 \mid \beta_{p} x_{p}\right)$, will be an estimate of the probability that the $p$ th document belongs to the category. The decision of whether to assign the category can be based on comparing the probability estimate with a threshold or by computing which decision gives optimal expected utility. 


\subsection{Interval-Valued Possibility Mean Value}

The concept of interval-valued possibility mean value are divided into two parts which are lower and upper possibility mean value. The lower $\underline{M}(\tilde{\tilde{A}})$ and upper $\bar{M}(\tilde{\tilde{A}})$ possibility mean value for interval type-2 fuzzy set are denoted as follow [16]:

$$
\begin{aligned}
& \underline{M}(\tilde{\tilde{A}})=\int_{0}^{h_{U}} \alpha\left(a_{1}^{U}+\frac{a_{2}^{U}-a_{1}^{U}}{h_{u}} \alpha\right) d \alpha+\int_{0}^{h_{L}} \beta\left(a_{1}^{L}+\frac{a_{2}^{L}-a_{1}^{L}}{h_{L}} \beta\right) d \beta \\
& \bar{M}(\tilde{\tilde{A}})=\int_{0}^{h_{U}} \alpha\left(a_{4}^{U}+\frac{a_{3}^{U}-a_{4}^{U}}{h_{u}} \alpha\right) d \alpha+\int_{0}^{h_{L}} \beta\left(a_{4}^{L}+\frac{a_{3}^{L}-a_{4}^{L}}{h_{L}} \beta\right) d \beta
\end{aligned}
$$

For crisp value, we can compute by using the average of lower and upper possibility mean value above that denoted as follows

$$
M(\tilde{\tilde{A}})=\frac{M+\bar{M}}{2}
$$

In this paper, the numerical analysis for proposed methodology is compared with interval-valued possibility mean value that proposed by [16].

\section{Proposed Method}

As noted in the introduction, the useful of interval type-2 fuzzy sets nowadays are widely applied in many research areas in dealing with uncertainty in data analysis which consistent with human intuition at the same time. A lot of researchers attempt to eliminate the need of human intuition in data analysis processes. Human intuition is strictly can't be eliminated because it can drive us towards uncertainty problems. This study simplify the concept of attributes to $\mu_{A \approx} \in[0,1]$ for fuzzy events. The values of attributes correspond to interval type-2 fuzzy sets. This study proposed a new centroid defuzzification method for Bayesian logistic regression algorithm. The methodology consist of two stages here namely:

\section{A. Stage one}

The development of an extension of the Vectorial Centroid defuzzification for interval type-2 fuzzy set.

Let consider by $\quad \tilde{A}_{i}=\left(\tilde{A}_{i}^{U}, \tilde{A}_{i}^{L}\right)=\left(\left(a_{i 1}^{U}, a_{i 2}^{U}, a_{i 3}^{U}, a_{i 4}^{U} ; H_{1}\left(\tilde{A}_{i}^{U}\right), \tilde{H}_{2}\left(\tilde{A}_{i}^{L}\right)\right)\right.$, $\left.\left(a_{i 1}^{L}, a_{i 2}^{L}, a_{i 3}^{L}, a_{i 4}^{L} ; H_{1}\left(\tilde{A}_{i}^{L}\right), H_{2}\left(\tilde{A}_{i}^{L}\right)\right)\right)$ as the interval type-2 fuzzy set. The complete method process of Vectorial Centroid is signified as follows: 
Step 1: Compute the centroid points of the three parts of $\alpha, \beta$ and $\gamma$ in interval type-2 fuzzy set representation as shown in Fig. 2.

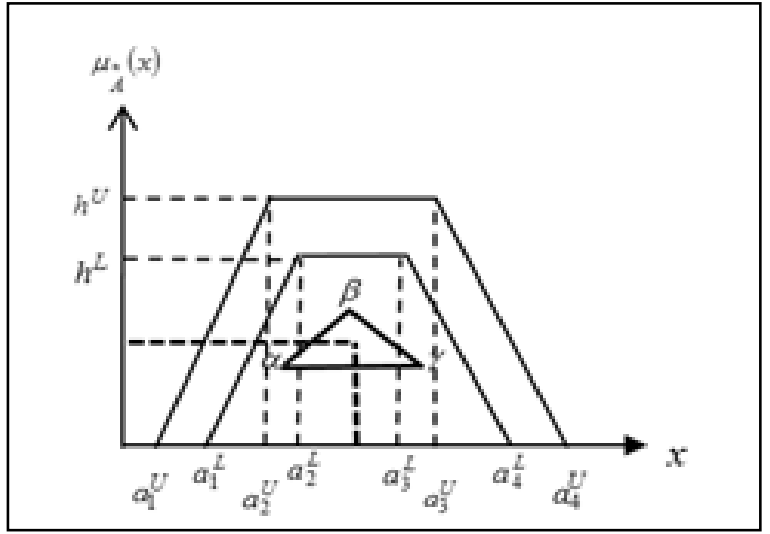

Fig. 2. Vectorial Centroid plane representation

$$
\begin{aligned}
& \alpha_{\bar{\alpha}, \underline{\alpha}}(x, y)=\left(\frac{1}{3}\left(a_{1}^{U}+\frac{1}{2} a_{2}^{U}\right)+\frac{1}{3}\left(a_{1}^{L}+\frac{1}{2} a_{2}^{L}\right), \frac{1}{6}\left(h^{U}+h^{L}\right)\right) \\
& \beta_{\bar{\beta}, \underline{\beta}}(x, y)=\left(\frac{1}{4}\left(a_{2}^{U}+a_{3}^{U}+a_{2}^{L}+a_{3}^{L}\right), \frac{1}{4}\left(h^{U}+h^{L}\right)\right) \\
& \gamma_{\bar{\gamma}, \underline{\gamma}}(x, y)=\left(\frac{1}{3}\left(a_{3}^{U}+\frac{1}{2} a_{4}^{U}\right)+\frac{1}{3}\left(a_{3}^{L}+\frac{1}{2} a_{4}^{L}\right), \frac{1}{6}\left(h^{U}+h^{L}\right)\right)
\end{aligned}
$$

Step 2: Connect all vertices centroids points of $\alpha, \beta$ and $\gamma$ each other, where it will generate another triangular plane inside of trapezoid plane.

Step 3: The centroid index of Vectorial Centroid of $(\tilde{x}, \tilde{y})$ with vertices $\alpha, \beta$ and $\gamma$ can be calculated as:

$$
\begin{aligned}
V C_{\tilde{A}}(\tilde{x}, \tilde{y}) & =\left(\frac{\alpha_{\bar{\alpha}, \underline{\alpha}}(x, y)+\beta_{\bar{\beta}, \underline{\beta}}(x, y)+\gamma_{\bar{\gamma}, \underline{\gamma}}(x, y)}{3},\right. \\
\beta_{\bar{\beta}, \underline{\beta}}(x, y)+ & {\left.\left[\frac{2}{3}\left(\frac{\alpha_{\bar{\alpha}, \underline{\alpha}}(x, y)+\gamma_{\bar{\gamma}, \underline{\gamma}}(x, y)}{2}-\beta_{\bar{\beta}, \underline{\beta}}(x, y)\right)\right]\right) }
\end{aligned}
$$

Vectorial Centroid can be summarised as 


$$
\begin{aligned}
V C_{\tilde{A}}(\tilde{x}, \tilde{y})= & \left(\frac { 1 } { 9 } \left[a_{1}^{U}+a_{1}^{L}+\frac{5}{4}\left(a_{2}^{U}+a_{2}^{L}\right)+\frac{7}{4}\left(a_{3}^{U}+a_{3}^{L}\right)+\right.\right. \\
& \left.\left.\frac{1}{2}\left(a_{4}^{U}+a_{4}^{L}\right)\right], \frac{11}{36}\left(h^{U}+h^{L}\right)\right)
\end{aligned}
$$

where

$$
\begin{aligned}
& \alpha: \text { the centroid coordinate of first triangle plane } \\
& \beta: \text { the centroid coordinate of rectangle plane } \\
& \gamma: \text { the centroid coordinate of second triangle plane }
\end{aligned}
$$

$(\tilde{x}, \tilde{y})$ : the centroid coordinate of fuzzy number $\tilde{\tilde{A}}$

Centroid index of Vectorial Centroid can be generated using Euclidean distance by [24]:

$$
R(\tilde{\tilde{A}})=\sqrt{\tilde{x}^{2}+\tilde{y}^{2}}
$$

B. Stage two

The implementation of Vectorial Centroid in Bayesian logistic regression.

Integrating fuzzy sets with Bayesian logistic regression in fuzzy states of nature, where if there is fuzzy dataset, defuzzification process is needed in converting into crisp values where at the same time the fuzzy nature is not lost. Reinterpretation of degree $\mu_{\tilde{\tilde{A}}} \in[0,1]$ using Vectorial Centroid to the $P\left(y=1 \mid \beta_{p} X_{p}\right)$ is developed as follows:

Step 1: Lift the reintergration of the fuzzy values membership function using trapezoidal interval type-2 fuzzy sets. Vectorial Centroid formulation are applied for trapezoidal interval type-2 fuzzy set rule formula. The $\mu_{\tilde{A}}$ represents as $\tilde{\tilde{A}}_{1}=\left(\tilde{A}_{1}^{U}, \tilde{A}_{1}^{L}\right)=\left(\left(a_{11}^{U}, a_{12}^{U}, a_{13}^{U}, a_{14}^{U} ; H_{1}\left(\tilde{A}_{1}^{U}\right), H_{2}\left(\tilde{A}_{1}^{U}\right)\right),\left(a_{11}^{L}, a_{12}^{L}, a_{14}^{L} ; H_{1}\left(\tilde{A}_{1}^{L}\right), H_{2}\left(\tilde{A}_{1}^{L}\right)\right)\right)$ in calculation to avoid cluttering.

Step 2: The centroid index of Vectorial Centroid, $R(\tilde{\tilde{A}})$ is inserted into Bayesian logistic regression rule as

$$
R(\tilde{\tilde{A}})=\sqrt{\tilde{x}^{2}+\tilde{y}^{2}}=\mu(\tilde{\tilde{A}})
$$

The computational process of likelihood and posterior distribution of fuzzy Bayesian logistic regression using Vectorial Centroid are denoted as 


$$
\begin{aligned}
\text { likelihood }_{i}= & \prod_{i=1}^{n}\left[\left(\frac{e^{\beta_{0}+\beta_{1} \mu(\tilde{\tilde{A}})_{i 1}+\ldots+\beta_{p} \mu(\tilde{A})_{i p}}}{1+e^{\beta_{0}+\beta_{1} \mu(\tilde{\tilde{A}})_{i 1}+\ldots+\beta_{p} \mu(\tilde{A})_{i p}}}\right)^{y_{i}}\left(1-\frac{e^{\beta_{0}+\beta_{1} \mu(\tilde{A})_{i 1}+\ldots+\beta_{p} \mu(\tilde{A})_{i p}}}{1+e^{\beta_{0}+\beta_{1} \mu(\tilde{\tilde{A}})_{i 1}+\ldots+\beta_{p} \mu(\tilde{\tilde{A}})_{i p}}}\right)^{\left(1-y_{i}\right)}\right] \\
\text { Posterior= }= & \left.\prod_{i=1}^{n}\left[\left(\frac{e^{\beta_{0}+\beta_{1} \mu(\tilde{\tilde{A}})_{i 1}+\ldots+\beta_{p} \mu(\tilde{\tilde{A}})_{i p}}}{1+e^{\beta_{0}+\beta_{1} \mu(\tilde{A})_{i 1}+\ldots+\beta_{p} \mu(\tilde{A})}}\right)^{y_{i p}}\right)^{y_{i}}\left(1-\frac{e^{\beta_{0}+\beta_{1} \mu(\tilde{\tilde{A}})_{i 1}+\ldots+\beta_{p} \mu(\tilde{A})_{i p}}}{1+e^{\beta_{0}+\beta_{1} \mu(\tilde{A})_{i 1}+\ldots+\beta_{p} \mu(\tilde{A})_{i p}}}\right)^{\left(1-y_{i}\right)}\right] \\
& \times \coprod_{j=0}^{p} \frac{1}{\sqrt{2 \pi \sigma_{j}}} \exp \left\{-\frac{1}{2}\left(\frac{\beta_{j}-c_{j}}{\sigma_{j}}\right)^{2}\right\}
\end{aligned}
$$

\section{Experimental Settings}

The experiment is conducted using 10-fold cross validation on BUPA liver-disorder dataset from UCI machine learning repository [23] is used where donated by BUPA Medical Research Ltd. This liver-disorder classification dataset has 345 examples, 7 attributes and binary classes for dependent attribute. The first 5 attributes are measurements taken by blood tests that are thought to be sensitive to liver-disorders and might arise from excessive alcohol consumption. The sixth attribute is a sort of selector attribute where the subjects are single male individuals. The seventh attribute shows a selector on the dataset which being used to split into two categories that indicating the class identity. The attributes include:
a. Mean corpuscular volume,
b. Alkaline phosphatase,
c. Aspartate aminotransferase,
d. Gamma-glutamyl transpeptidase,
e. Alamine aminotransferase,
f. Number if half-pint equivalents of alcoholic beverage drunk per day, and
g. Output attributes either liver disorder or liver normal

Among all the people, there are 145 belonging to the liver-disorder group and 200 belonging to the liver-normal group. These attributes are selected with the aid of experts. The original dataset are fuzzified randomly in interval type-2 fuzzy sets form in operating centroid methods. Suppose that attribute alkaline phosphatase, aspartate aminotransferase, gamma-glutamyl transpeptidase and alamine aminotransferase are fuzzy events, $\mu_{\beta_{i} x_{i}}$. Below describes the example of interval type-2 fuzzy sets are used in this research study: 
Example: If the trapezoidal interval type-2 fuzzy set $\tilde{\widetilde{A}}_{i}=\left(\tilde{A}_{i}^{U}, \tilde{A}_{i}^{L}\right)=((15.35,16.68,18.06,20.51 ; 1),(16,17,18,19 ; 0.9))$, then the centre points are computed using proposed of extension Vectorial Centroid and intervalvalued possibility mean value [16] formulation respectively as follows:

Vectorial Centroid:

$V C(x)=17.3678$ and $V C(y)=0.58056$

Centroid index Vectorial Centroid, $V C(\stackrel{\widetilde{R}}{R})=17.3775$

Interval-Valued Possibility Mean Value:

$M(\tilde{\tilde{A}})=\left[M_{*}(\tilde{\tilde{A}}), M^{*}(\tilde{\tilde{A}})\right]=[14.8683,16.8633]$

Crisp possibility mean value, $M(\widetilde{\widetilde{A}})=15.8658$

\section{Simulation Results}

This section illustrates the validation process of the methodology in theoretically and empirically. Therefore, the theoretical of Vectorial Centroid validation process are as follow:

\section{A. Stage one}

The relevant properties considered for qualifying the applicability of centroid for interval type-2 fuzzy set, where they depend on the practicality within the area of research however, they are not considered as complete. Therefore, without loss of generality, the relevant properties of the centroid are as follow:

Let $\tilde{A}$ and $\tilde{B}$ are be trapezoidal and triangular interval type-2 fuzzy set respectively, while $V C_{\tilde{\tilde{A}}}(\tilde{x}, \tilde{y})$ and $V C_{\tilde{B}}(\tilde{x}, \tilde{y})$ be centroid points for $\tilde{A}$ and $\tilde{B}$ respectively. Centroid index of Vectorial Centroid, $(R)$ shows the crisp value of centroid point that is denoted as $R(\tilde{A})=\sqrt{\tilde{x}^{2}+\tilde{y}^{2}}$.

Property 1: If $\tilde{\tilde{A}}$ and $\tilde{\tilde{B}}$ are embedded and symmetry, then $R(\tilde{\tilde{A}})>R(\tilde{\widetilde{B}})$. Proof:

Since $\tilde{\tilde{A}}$ and $\tilde{B}$ are embedded and symmetry, hence from equation (15) we have $\sqrt{\tilde{x}_{\tilde{\tilde{A}}}^{2}+\tilde{y}_{\tilde{\tilde{A}}}^{2}}>\sqrt{\tilde{x}_{\tilde{B}}^{2}+\tilde{y}_{\tilde{B}}^{2}}$. Therefore, $R(\tilde{\tilde{A}})>R(\tilde{B})$. 
Property 2: If $\tilde{\tilde{A}}$ and $\tilde{\tilde{B}}$ are embedded with $\left(h^{U}, h^{L}\right)_{\tilde{A}}>\left(h^{U}, h^{L}\right)_{\tilde{B}}$, then $R(\tilde{A})>R(\tilde{B})$.

\section{Proof:}

Since $\tilde{\tilde{A}}$ and $\tilde{\tilde{B}}$ are embedded and with $\left(h^{U}, h^{L}\right)_{\tilde{A}}\left(h^{U}, h^{L}\right)_{\tilde{B}}$, hence we know that $\tilde{y}_{\tilde{A}}>\tilde{y}_{\tilde{B}}$.

Then, from equation (15) we have $\sqrt{\tilde{x}_{\tilde{A}}^{2}+\tilde{y}_{\tilde{A}}^{2}}>\sqrt{\tilde{x}_{\tilde{B}}^{2}+\tilde{y}_{\tilde{B}}^{2}}$. Therefore, $R(\tilde{\tilde{A}})>R(\tilde{\tilde{B}})$.

Property 3: If $\tilde{\tilde{A}}$ is singleton fuzzy number, then $R(\tilde{\tilde{A}})=\sqrt{\tilde{x}_{\tilde{A}}^{2}+\tilde{y}_{\tilde{\tilde{A}}}^{2}}$.

Proof:

For any crisp (real) interval type-2 fuzzy set, we know that $a_{1}^{U}=a_{2}^{U}=a_{3}^{U}=a_{4}^{U}=a_{1}^{L}=a_{2}^{L}=a_{3}^{L}=a_{4}^{L}=\tilde{x}_{\tilde{\mathrm{A}}}$ which are equivalent to equation (14). Therefore, $R(\tilde{\tilde{A}})=\sqrt{\tilde{x}_{\tilde{A}}^{2}+\tilde{y}_{\tilde{A}}^{2}}$.

Property 4: If $\tilde{\tilde{A}}$ is any symmetrical or asymmetrical interval type-2 fuzzy number, then $a_{1}^{U}<R(\tilde{\tilde{A}})<a_{4}^{U}$

Proof:

Since any symmetrical or asymmetrical interval type-2 fuzzy set has $a_{1}^{U} \leq a_{2}^{U} \leq a_{3}^{U} \leq a_{4}^{U}$, hence $a_{1}^{U}<V C_{\tilde{A}}(\tilde{x}, \tilde{y})<a_{4}^{U}$. Therefore, $a_{1}^{U}<R(\tilde{\tilde{A}})<a_{4}^{U}$.

\section{$B$ Stage two}

Aforementioned, the empirical validation is implemented where the BUPA liverdisorder data set is used in conducting Bayesian Logistic Regression data analysis.

Note that this study is considered all type of possible interval type-2 fuzzy set for attributes randomly as figures follow: 


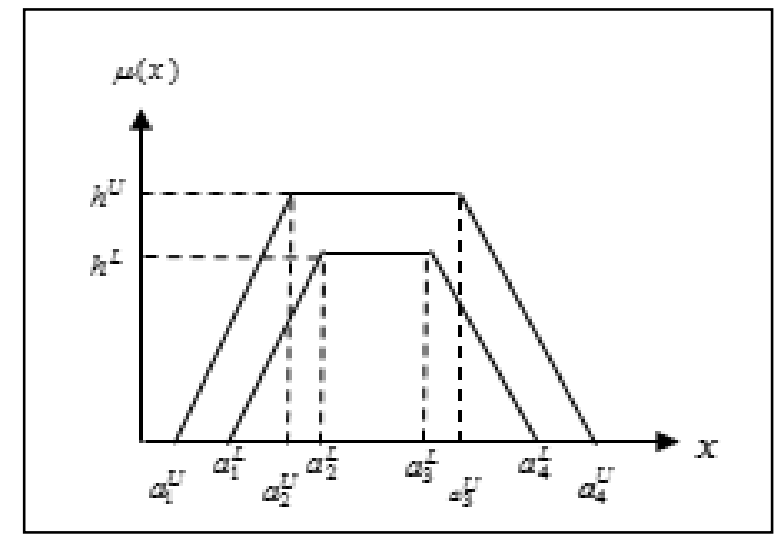

Fig. 3. Trapezoidal non-normal symmetry

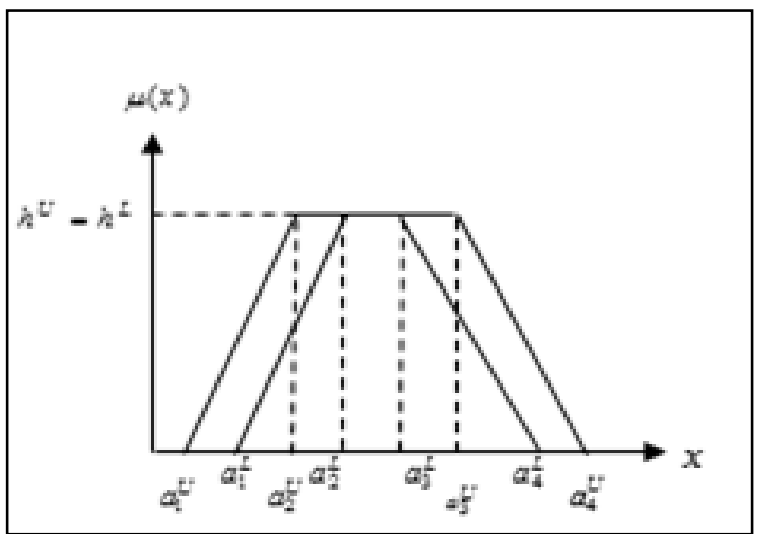

Fig. 4. Trapezoidal normal symmetry

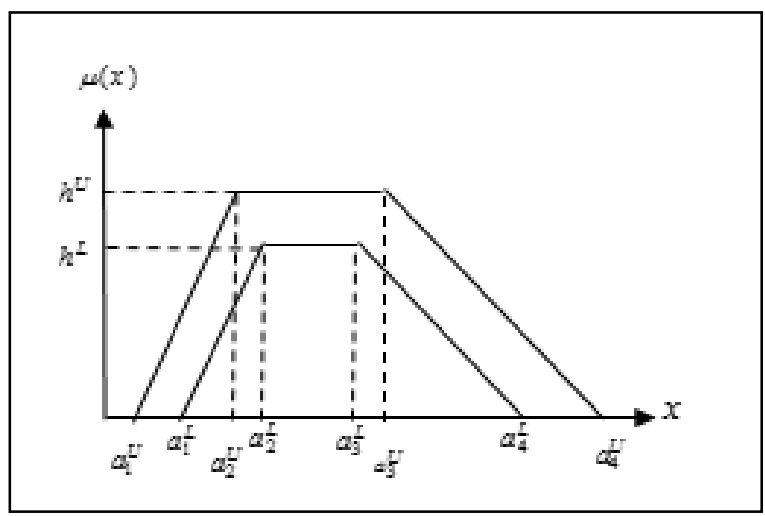

Fig. 5. Trapezoidal non-normal asymmetry 


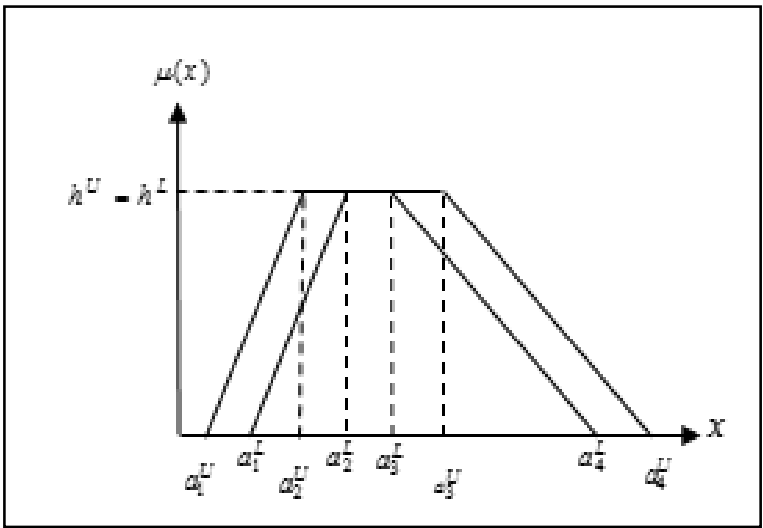

Fig. 6. Trapezoidal normal asymmetry

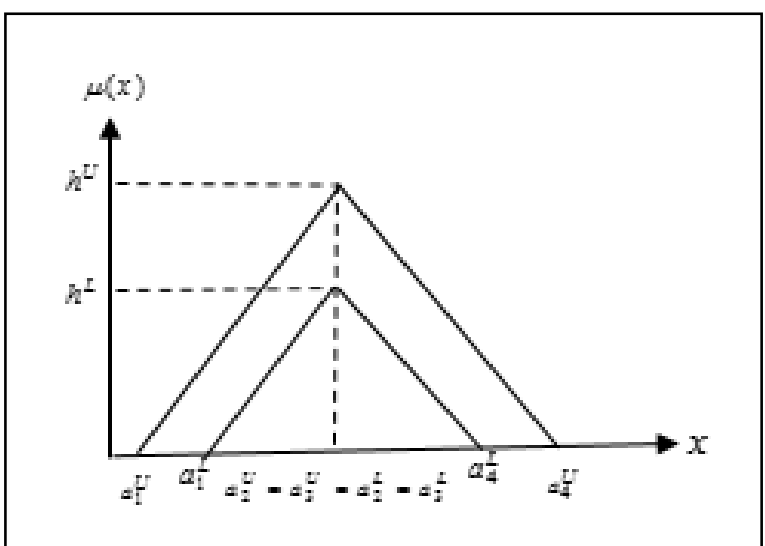

Fig. 7. Triangular non-normal symmetry

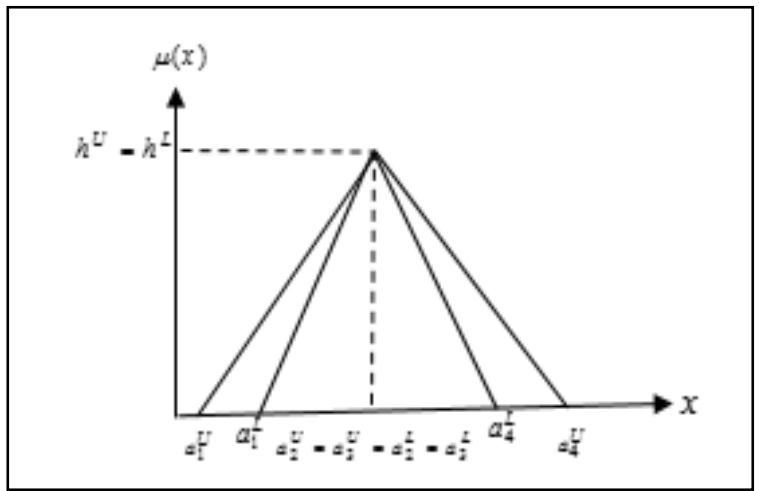

Fig. 8. Triangular normal symmetry 


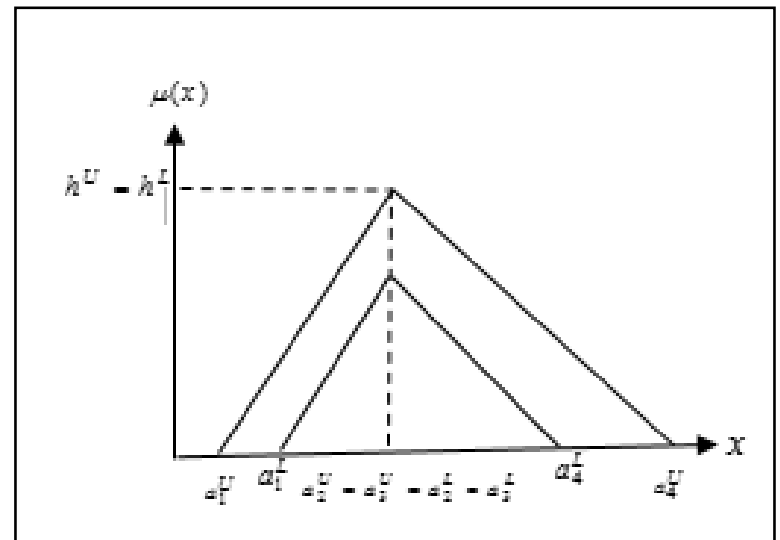

Fig. 9. Triangular non-normal asymmetry

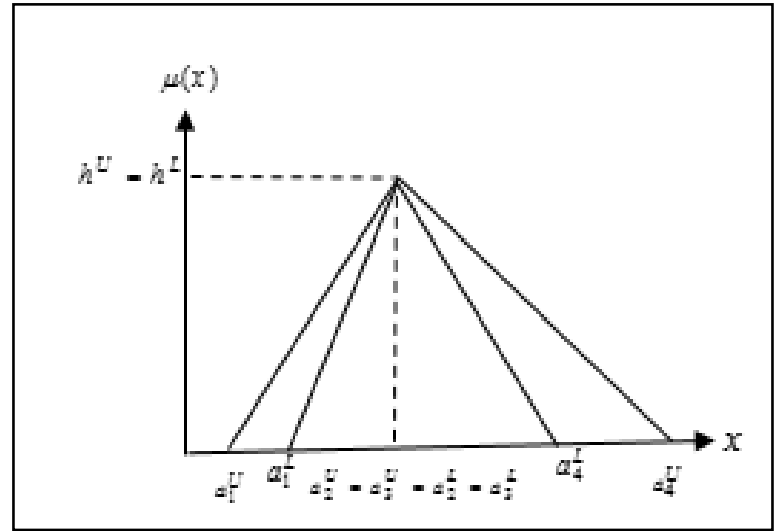

Fig. 10. Triangular normal asymmetry

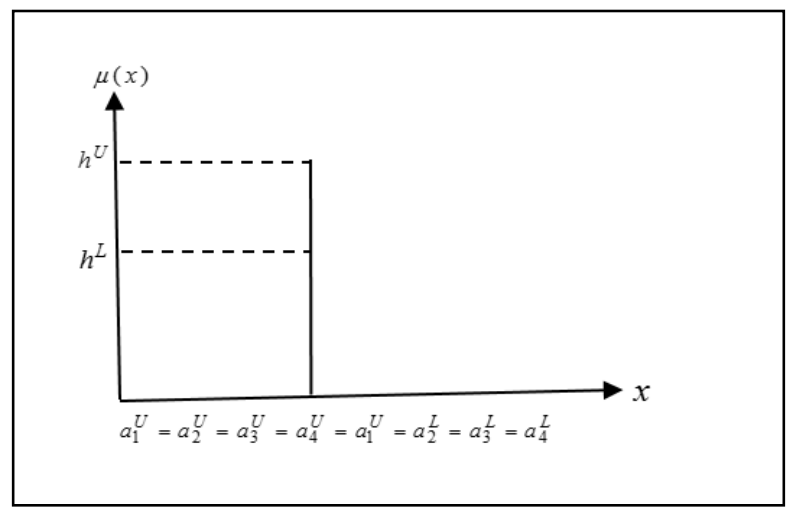

Fig. 11. Singleton non-normal 


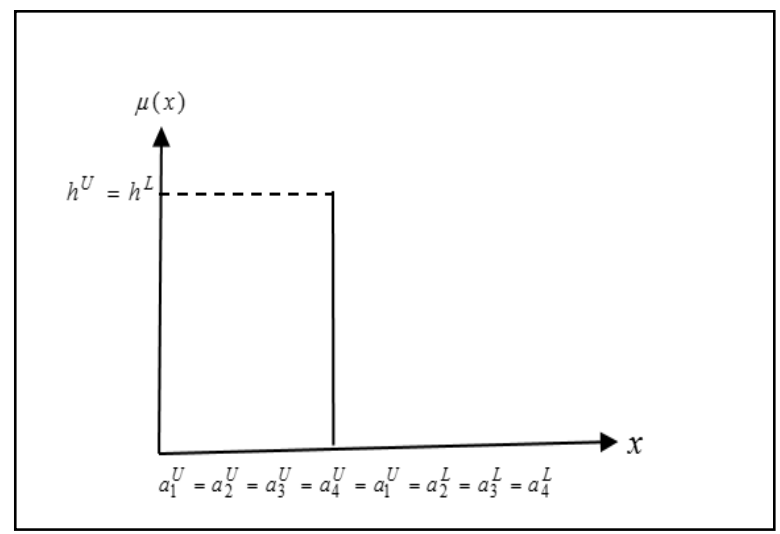

Fig. 12. Singleton Normal

In simulation analysis, the data set is randomly partitioned into 10 equal sized partitions. Then, one of the partitions is used to test, while the rest of the partitions is dedicated to train the base learner. This procedure is repeated ten times so that each partition is used for the test exactly one time. Here, a mean accuracy of the individual results is combined. Table 1 presents a comparative results between classical Bayesian logistic regression (BLR-Classic), Bayesian logistic regression using possibility mean value [16] method (BLR-PMV), and Bayesian logistic regression using the extension of Vectorial Centroid (BLR-VC). The comparison results are based on accuracy, precision, sensitivity, specificity, Kappa statistic, and some error terms: Mean Absolute Error (MAE), Root Mean Square Error (RMSE), Relative Absolute Error (RAE) and Root Relative Square Error (RRSE).

Table 1. Accuracy, precision, sensitivity, specificity Kappa statistic and error terms.

\begin{tabular}{lccc}
\hline Method & BLR-Classic & BLR-PMV & BLR-VC \\
\hline Accuracy & $67.2464 \%$ & $58.5507 \%$ & $68.1159 \%$ \\
Precision & $17.67 \%$ & $1.4 \%$ & $30.34 \%$ \\
Sensitivity & $82 \%$ & $66.67 \%$ & $83.02 \%$ \\
Specificity & $64.75 \%$ & $58.41 \%$ & $65.41 \%$ \\
Kappa Statistic & 0.2613 & 0.0203 & 0.2832 \\
Error: & & & \\
MAE & 0.3275 & 0.4145 & 0.3188 \\
$\boldsymbol{R} \boldsymbol{M S E}$ & 0.5723 & $0.6438 \%$ & 0.5647 \\
$\boldsymbol{R} \boldsymbol{A} \boldsymbol{E}$ & $67.2025 \%$ & $85.0438 \%$ & $65.4183 \%$ \\
$\boldsymbol{R} \boldsymbol{R S E}$ & $115.9404 \%$ & $130.4259 \%$ & $114.391 \%$ \\
\hline
\end{tabular}

The accuracy and precision of a measurement system plays important role in quantifying the actual measure value. It is commonly used as metric for evaluation of machine learning systems. In addition to classification, accuracy results obtained by the algorithm. The precision is dependent of accuracy where the model can be very precise but inaccurate. The higher the value of accuracy and precision, the better classification prediction is made. In this research study, Table 1 shows the 
classification accuracy results that show the correctness of a model classifies the dataset in each class. Below shows the formulation of accuracy and precision:

$$
\begin{aligned}
& \text { Accuracy }: \frac{\text { TotalPositive }+ \text { TotalNegative }}{\text { Positive }+ \text { Negative }} \\
& \text { Precision }: \frac{\text { TotalPositive }}{\text { TotalPositive }+ \text { FalsePositive }}
\end{aligned}
$$

The classification accuracy results of BLR-Classic, BLR-PMV and BLR-VC are $67.2464 \%, 58.5507 \%$ and $68.1159 \%$ respectively. It shows that the proposed methodology is significantly more accurate and very promising compared to others. The highest precision in this case study is BLR-VC with $30.34 \%$, followed by BLRClassic with $17.67 \%$ and BLR-PMV with $1.4 \%$. Precision discusses the closeness of two or more measurements to each other.

The sensitivity test mentions to the ability of the test to correctly identify those observers with positive predictive value. A high sensitivity is clearly imperative where the test is used to identify the correct class. But, specificity test is inversely proportional to sensitivity where it has the ability of the test to correctly identify those observers with negative predictive value [25]. Below are formulation to calculate sensitivity and specificity:

$$
\begin{aligned}
& \text { Sensitivity: } \frac{\text { TotalPositive }}{\text { TotalPositive }+ \text { FalseNegative }} \\
& \text { Specificity }: \frac{\text { TotalNegative }}{\text { FalsePositive }+ \text { TotalNegative }}
\end{aligned}
$$

It is interesting to observe that the proposed method, BLR-VC produces the highest sensitivity and specificity value with $83.02 \%$ and $65.41 \%$ respectively. The results for BLR-PMV shows the lowest results for sensitivity and specificity with $66.67 \%$ and $58.41 \%$ respectively. It depicts that the goodness of prediction of both tests for BLRPMV is lesser than BLR-Classic and BLR-VC.

Kappa statistic technique is used to measure the agreement of two classifiers and estimate the probability of two classifiers agree simply by chance [26]. Known as chance-corrected measure of agreement between classification and the true classes, it is an evaluation metric which is based on the difference between the actual agreement in the error matrix and the chance agreement. The values for Kappa range from 0 to 1 and the higher the value of Kappa statistic, the stronger the strength of agreement between two classifiers by chance.

$$
\text { KappaStat, } k=\frac{p_{o}-p_{e}}{1-p_{e}}
$$


where

$$
\begin{aligned}
& p_{o} \text { is relative observed agreement among raters, } \\
& p_{e} \text { is the hypothetical probability of chance agreement. }
\end{aligned}
$$

BLR-VC shows the highest value of Kappa statistic with 0.2832 followed by BLRClassic and BLR-PMV with 0.2613 and 0.0203 respectively. It seems that, the proposed methodology produced strong agreement between two classifiers by chance.

The final part in Table I depicts the errors for the experiment carried out. The errors are computed using Mean Absolute Error (MAE), Root Mean Square Error (RMSE), Relative Absolute Error (RAE) and Root Relative Square Error (RRSE). All the statistic error terms compare true values to theirs estimates, but do it in a slightly different way. Below depict the formulation in calculating MAE, RMSE, RAE and RRSE:

$$
\begin{aligned}
& \text { MAE }=\frac{1}{N} \sum_{i=1}^{N}\left|\hat{\theta}_{i}-\theta_{i}\right| \\
& R M S E=\sqrt{\frac{1}{N} \sum_{i=1}^{N}\left(\hat{\theta}_{i}-\theta_{i}\right)^{2}} \\
& R A E=\frac{\sum_{i=1}^{N}\left|\hat{\theta}_{i}-\theta_{i}\right|}{\sum_{i=1}^{N}\left|\bar{\theta}_{i}-\theta_{i}\right|} \\
& R R S E=\sqrt{\frac{\sum_{i=1}^{N}\left(\hat{\theta}_{i}-\theta_{i}\right)^{2}}{\sum_{i=1}^{N}\left(\bar{\theta}_{i}-\theta_{i}\right)^{2}}}
\end{aligned}
$$

These error terms demonstrates how disperse away the estimated values from the true value of $\theta$. MAE and RMSE calculate the average difference between those two values. For every data point, the distance is take vertically from the point of the corresponding estimated value on the curve fit and square the value. RMSE is directly interpretable in terms of measurement units that measure of goodness of fit. In RAE and RRSE, we divide those differences by the variation of $\theta$ where they have a scale from 0 to 1 , then we would multiply those value by 100 to get the similarity in $0-100$ scale. In this simulation results, the proposed methodology, BLR-MC performs better in error terms where all of these errors are less than BLR-Classic and BLR-PMV. 


\section{Conclusion}

The usefulness of fuzzy Bayesian knowledge in understanding and modelling complex uncertainty associated with real-world problems is presented in this paper. This research study has carried out an extension based Vectorial Centroid for interval type-2 fuzzy set with Bayesian logistic regression. Bayesian logistic regression algorithm that takes into account the need of fuzzy events in attributes. This work consist of two stages which are: (1) The development of Vectorial Centroid defuzzification method for interval type-2 fuzzy set and: (2) The implementation of Vectorial Centroid in Bayesian logistic regression. For the primary stage, the development of new centroid method can cater all the possible cases of interval type-2 fuzzy set precisely that matching for human intuition. The implementation in Bayesian logistic regression using proposed method on stage two is easily capable constructed and handled in data analysis when dealing with fuzzy data sets. The contribution of the paper can be summarised as follows. First, the development of new defuzzification method which can cater all possible cases in interval type-2 fuzzy sets and considering human judgment or intuition. Second, the presented hybrid intelligent classification model utilised the consistency-based feature selection between new Vectorial Centroid defuzzification method and Bayesian logistic regression model. The experimental results on Table 1 indicates that the proposed hybrid classification model can obtain very promising results in terms of accuracy, precision, sensitivity, specificity, Kappa statistics and error terms.

Although the model proposed in this paper is relatively simple conceptually, some drawbacks may exist in this research study. First, the proposed hybrid classification model for interval type-2 fuzzy numbers was developed and tested on BUPA liverdisorder dataset from WEKA (Waikato Environment for Knowledge Analysis) software. The useful of interval type-2 fuzzy set for attributes are randomly computed. Hence, the implementation or development of special linguistic terms for attributes are needed for remarkable outputs. Second, the scope of this research study is focused to be automated diagnosis liver-disorder. Still, more experimental work should be enthusiastic to obtain a medical classification model with a better ability of generalization under fuzzy environment. Finally, the proposed Vectorial Centroid only applied for Bayesian logistic regression. It should be applied and compared with other machine learning systems in the future work that would make research much more convincing.

Despite the above drawbacks, this study can be profitable alternatively in the set of existing Bayesian logistic regression algorithms for various problems in machine learning such as inference, classification, clustering, regression and so forth. There are four relevant properties for centroid development are constructed and well proved in theoretical validation, where corresponding with all possible interval type-2 fuzzy set representation. Several tests for validation have been done and the results have been studied in-depth using BUPA liver-disorder classification dataset from UCI machine learning repository. With this promising preliminary simulation results, the proposed research study presents more effective in dealing with fuzzy events empirically. To conclude, the main focus of this research study can be continued in order to make some contributions by considering real case study drawn for diverse fields crossing ecology, health, genetics, finance and so forth. To take care of the hybrid of fuzzy 
machine learning systems, a more general concepts of general fuzzy numbers and fuzzy vectors along the characterising function must be applied in capturing the imprecision and uncertainty in data analysis.

\section{References}

1. Tang, Y., Pan, H., Xu, Y.: Fuzzy Naïve Bayes Classifier Base on Fuzzy Clustering. In: IEEE International Conference on Systems, Mans and Cybernatics, IEEE International Conference, vol. 5, pp. 6pp. IEEE Press (2002)

2. Klir, G., Yuan, B.: Fuzzy Sets and Fuzzy Logic: Theory and Application. In: Prentice Hall, Upper Saddle River (1995)

3. Wagner, C., Hargas, H.: Uncertainty and Type-2 Fuzzy Sets Systems. In: IEEE UK Workshop on Computational Intelligent (UKCI) (2010)

4. Zadeh, L.A.: Fuzzy Sets. In: J. Info. \& Contr. 8, 338--353 (1965)

5. Deng, H.: Comparing and Ranking Fuzzy Numbers Using Ideal Solutions. J. App. Math. Mod. 38, 1638--1646 (2013)

6. Zimmerman, H.J.: An Application - Oriented View of Modelling Uncertainty. Euro. J. Oper. Res. 122, 190-198 (2000)

7. Hullermeire, E.: Fuzzy Sets in Machine Learning and Data Mining. J. App. Soft Comp. 11, $1493-1505$ (2011)

8. Zadeh, L.A.: The Concept of a Linguistic Variable and Its Application to Approximate Reasoning. J. Info. Sci. 8, 199-249 (1975)

9. Karnik, N.N., Mendel, J.M.: Centroid of Type-2 Fuzzy Set. J. Info. Sci. 132, 195-220 (2001)

10. Mendel, J.M., John, R.I., Liu, F.L.: Interval Type-2 Fuzzy Logical Systems Made Simple. J. Fuz. Sys. IEEE Trans. 14, 808-821 (2006)

11. Wallsten, T.S., Budescu, D.V.: A Review of Human Linguistic Probability Processing General Principles amd Empirical Evidences. J. The Know. Eng. Rev. 10:1, 43 - 62 (1995)

12. Yager, R.R., Filev, D.P.: Essential of Fuzzy Modelling and Control. Wiley, New York (1994)

13. Mogharreban, N., Dilalla, L.F.: Comparison of Defuzzification Techniques for Analysis of Non-Interval Data. In: Fuzzy Information Processing Society, NAFIPS 2006. Annual Meeting of the North American, pp. 257-260 (2006)

14. Mendel, J.M.: Uncertain Rule-Based Fuzzy Logic Systems: Introduction and New Directions. Prentice-Hall, Upper Saddle River, New Jersey (2001)

15. Liu, F.: An Effect Centroid Type-Reduction Strategy for General Type-2 Fuzzy Logic System. J. Info. Sci. 178, 2224-2236 (2008)

16. Gong, Y., Hu, N., Zhang, J., Liu, G., Deng, J.: Multi-Attribute Group Decision Making Method Based on Geometric Bonferroni Mean Operator of Trapezoidal Interval Type-2 Fuzzy Numbers. J. Comp. \& Ind. Eng. 81, 167-176 (2015)

17. Carlsson, C., Fuller, R.: On Possibility Mean Value and Variance of Fuzzy Numbers. J. Fuz. Sets \& Sys. 122, 315-326 (2001)

18. Ku Khalif, K.M.N., Gegov, A.:Generalised Fuzzy Bayesian Network with Adaptive Vectorial Centroid. In: $16^{\text {th }}$ World Congress of the International Fuzzy Systems Association (IFSA) \& $9^{\text {th }}$ Conference of European Society for Fuzzy Logic and Technology (EUSFLAT), pp. 757-764. Atlantis Press, Gijon, Spain (2015)

19. Chen, C.C.M., Schwender, H., Keith, J., Nunkesser, R., Mengersen, K., Macrossan, P.: Method of Identifying SNP Interactions: A Review on Variations of Logic Regrssion, Random Forest and Bayesian Logistic Regression. IEEE/ ACM Transactions. Comp. Bio. \& Bioinfo.. 8, 1580-1591 (2011) 
20. Choy, S.L.: Priors: Silent or Active Partners of Bayesian Inference?. Case Studies in Bayesian Statistical Modelling and Analysis. John Wiley \& Sons Ltd, Sussex (2013)

21. Lee, L.W., Chen, S.M.: Fuzzy Attributes Group Decision-Making Based on the Extension of TOPSIS Method and Interval Type-2 Fuzzy Sets. In: $7^{\text {th }}$ IEEE International Conference on Machine Learning and Cybernatics, pp. 3260-3265, IEEE Press, Kunming (2008)

22. Bayesian Inference for Logistic Regression Parameters,

http://www.medicine.mcgill.ca/epidemiology/joseph/courses/EPIB-621/bayeslogit.pdf

23. Liver Disorder Data Set, UCI Machine Learning Repository,

https://archive.ics.uci.edu/ml/datasets/Liver+Disorders

24. Cheng, C.H.: A New Approach for Ranking Fuzzy Numbers by Distance Method. J. Fuz. Set. \& Sys, 95, 307-317 (1998)

25. Clinical Tests: Sensitivity and Specificity, http://ceaccp.oxfordjournals.org/content/8/6/221.full

26. Jeong, D., Kang, D., Won, S.: Feature Selection for Steel Defects Classification. In: International Conference on Control Automation and Systems (ICCAS), pp. 338-341. IEEE Press, Gyeonngi-do (2010) 